\section{FRI0335 EARLIER ONSET AND BRAINSTEM INVOLVEMENT AS KEY FEATURES IN A BRAZILIAN NEURO-BEHÇET'S DISEASE COHORT}

L.L. Prado ${ }^{1}$, G. Tinone ${ }^{2}$, D. Callegaro ${ }^{2}$, C.G.S. Saad ${ }^{1}$, M. Levy-Neto ${ }^{1}$ C. Goncalves ${ }^{1} .{ }^{1}$ Rheumatology Division; ${ }^{2}$ Neurology Division, Faculdade de Medicina Da Universidade de Sao Paulo, São Paulo, Brazil

Background: Behçet's disease (BD) is a multisystem disease in which central nervous system involvement - neuro-Behçet's disease (NBD) - may strike young patients with devastating consequences. In this regard, early diagnosis and treatment is essential to prevent injury.

Objectives: This study aimed to analyze NBD clinical features compared to non-neurological $\mathrm{BD}$ in order to distinguish disease patterns.

Methods: A retrospective study was performed in $101 \mathrm{BD}$ outpatients from a single tertiary center followed between 2011 and 2016. BD diagnosis was based on the 2014 International Criteria for Behçet's Disease. Demographic, clinical and imaging features of 28 NBD patients were compared to 73 BD patients without neurological involvement.

Results: Earlier disease onset was found in NBD compared to BD (26.0 \pm 10.2 vs. $30.2 \pm 8.8$ years, $p=0.04)$. There were no differences between genders incidences, with a female predominance in both groups (64.3 vs. $72.6 \%, p>0.05)$. Over half of patients $(53.6 \%$ ) presented NBD as the first symptom and the mean time between diagnosis and NBD onset was $3.8 \pm 5.9$ years. Uveitis was less frequent in NBD patients $(25 \%$ vs. $47.9 \%, p=0.04)$, together with cutaneous disease ( $50 \%$ vs. $76.7 \%, p=0.01)$ and articular involvement $(17.9 \%$ vs. $46.5 \%, \mathrm{p}=0.01)$. Oral ulcers, genital ulcers, intestinal and vascular involvement frequencies were similar in both groups. Regarding NBD presentation, brainstem involvement was the most prevalent $(67.9 \%)$, followed by central venous thrombosis $(32.1 \%)$, aseptic meningitis (17.9\%), stroke (3.6\%) and peripheral neuropathy (3.6\%). Most patients $(82.1 \%)$ had a single neurological attack whereas relapsing disease was found in $18.5 \%$.

Conclusions: Our study found an earlier disease onset in NBD patients and a lower frequency of ocular, cutaneous and articular involvement. Moreover, several patients may unfold the disease as NBD, with lack of other manifestations. In addition, brainstem lesions occurred in most patients. Recognizing these disease patterns might support to expedite NBD diagnosis.

Disclosure of Interest: None declared

DOI: 10.1136/annrheumdis-2017-eular.5514

\section{FRI0336 EFFICACY OF TOCILIZUMAB IN 31 PATIENTS WITH GIANT CELL ARTERITIS}

L.C. Domínguez-Casas ${ }^{1}$, J. Loricera ${ }^{1}$, J.L. Hernández ${ }^{2}$, S. Castañeda ${ }^{3}$, A. Mera ${ }^{4}$, E. Pérez-Pampín ${ }^{4}$, E. Peiró ${ }^{1}$, A. Humbría ${ }^{3}$, J. Calvo-Alén ${ }^{5}$, E. Aurrecoechea ${ }^{5}$, J. Narváez ${ }^{6}$, A. Sánchez-Andrade ${ }^{7}$, P. Vela ${ }^{8}$, E. Díez ${ }^{9}$, C. Moriano ${ }^{9}$, P. Lluch ${ }^{10}$, C. Moll ${ }^{10}$, Í. Hernández ${ }^{11}$, V. Calvo-Río ${ }^{1}$, J. Ivorra ${ }^{12}$ A. Corrales ${ }^{1}$, F. Ortiz-Sanjuán ${ }^{12}$, C. González-Vela ${ }^{1}$, M.Á. González-Gay ${ }^{1}$, R. Blanco ${ }^{1} \cdot{ }^{1}$ Rheumatology; ${ }^{2}$ Internal Medicine, Hospital Universitario Marqués de Valdecilla. IDIVAL, Santander; ${ }^{3}$ Rheumatology, Hospital Universitario de la Princesa, IIS-IP, Madrid; ${ }^{4}$ Rheumatology, Complejo Hospitalario Universitario de Santiago, Santiago de Compostela: ${ }^{5}$ Rheumatology, Hospital de Sierrallana, Torrelavega; ${ }^{6}$ Rheumatology, Hospital de Bellvitge, Barcelona: ${ }^{7}$ Rheumatology, Hospital Universitario Lucus Augusti, Lugo; ${ }^{8}$ Rheumatology, Hospital General Universitario de Alicante, Alicante; ${ }^{9}$ Rheumatology, Complejo Asistencial Universitario de León, León; ${ }^{10}$ Rheumatology, Hospital Mateu Orfila, Menorca;

${ }^{11}$ Rheumatology, Complexo Hospitalario Universitario de Vigo, Vigo;

${ }^{12}$ Rheumatology, Hospital Universitario y Politécnico la Fe, Valencia, Spain

Background: Giant cell arteritis (GCA) is often a disease refractory to corticosteroids and, besides, the efficacy of immunosuppressive agents is not well established. In recent years, several case reports and small case series have shown efficacy with the use of tocilizumab (TCZ).

Objectives: Our aim was to assess in a clinical practise setting the short and long-term efficacy of TCZ in GCA patients with refractory disease and/or with unacceptable side effects due to corticosteroids.

Methods: Retrospective multicenter open-label study on $31 \mathrm{GCA}$ patients treated with TCZ [intravenously at standard dose of $8 \mathrm{mg} / \mathrm{kg} / \mathrm{monthly}(\mathrm{n}=29)$, and subcutaneously at a dose of $162 \mathrm{mg} /$ week $(\mathrm{n}=2)]$. We assessed the efficacy on clinical and laboratory parameters, the reduction of the dose of corticosteroids, as well as the short and long-term side effects and the possibility of discontinuation or reduction of the dose of TCZ. Wilcoxon test was used to compare laboratory parameters across time.

Results: We included 31 patients (24 women/ 7 men), with a mean age of $73 \pm 9$ years. The main clinical features at TCZ onset were: polymyalgia rheumatica $(n=21)$, asthenia $(n=8)$, headache $(n=8)$, constitutional syndrome $(n=11)$, jaw claudication $(n=3)$, and visual loss $(n=4)$. Besides corticosteroids and before TCZ onset, 26 patients had also received several conventional immunosuppressive and/or biologic drugs. Twenty-seven of 31 patients achieved a rapid and maintained clinical improvement after TCZ therapy (Table). After a median follow-up of 18 [interquartile range, $6-30$ ] months we observe a reduction of the median of: a) CRP from $1.9[1.1-3.7]$ to $0.1[0.1-0.7] \mathrm{mg} / \mathrm{dL}$; b) ESR from 44 [17-74] to 12 [4-16] $\mathrm{mm} / 1 \mathrm{st}$ hour; and c) the dose of prednisone from 20 [10-45] to 2.5 [0-7.5] mg/day. In this follow-up period, the outcome of patients was as follows: a) discontinuation of TCZ $(n=8)$ due to sustained remission; $b$ ) dose reduction due to improvement $(n=5)$ or side effects $(n=2)$; c) withdrawal of TCZ because of side effects $(n=7)$; and d) the same dose that at onset $(n=8)$. The reasons why TCZ had to be discontinued were: severe neutropenia; recurrent pneumonia; colon adenocarcinoma; cytomegalovirus infection; hypertensive crisis during infusion; myelodysplastic syndrome; and overall health deterioration. The latter patient died because of stroke. Another patient also died after the second TCZ infusion due to stroke in the context of an infective endocarditis.

\begin{tabular}{|c|c|c|c|c|c|}
\hline & Bareline & Month 6 & Month 12 & Month 18 & Month 24 \\
\hline $\begin{array}{c}\text { Clinical improvement, \% } \\
\text { (n) }\end{array}$ & & $100^{x x}(27 / 27)$ & $95^{\mu \times}(2021)$ & $85^{m n}(11113)$ & $100^{\mathrm{xx}}(111 / 1)$ \\
\hline \multicolumn{6}{|l|}{$\begin{array}{c}\text { Laboratory markers } \\
\text { median [IQR] }\end{array}$} \\
\hline $\operatorname{ESR}\left(\mathrm{mm} / \mathrm{1}^{\mathrm{m}} \mathrm{h}\right)$ & $\begin{array}{c}44[177.74)^{* *} \\
(28)^{*}\end{array}$ & $\begin{array}{c}6\left[2-111^{1 \times *}\right. \\
(24)^{*}\end{array}$ & $\begin{array}{c}7.5[3-23]^{\infty} \\
(18)^{\infty}\end{array}$ & $\begin{array}{c}9.5[2-19)^{x} \\
(12)^{*}\end{array}$ & $\begin{array}{c}6[2-14]^{* * *} \\
(10)^{*}\end{array}$ \\
\hline CRP(mgdedl) & $\begin{array}{c}19[1.1-3.7)^{3 \times x} \\
(31)\end{array}$ & $\begin{array}{l}0.1[0.1-0.7]^{3 \times x} \\
(27]^{2}\end{array}$ & $\begin{array}{l}02[0.1-0.7]^{3 \times x} \\
(19)\end{array}$ & $\begin{array}{c}0.1[0.1-1.5]^{*} \\
(13)^{*}\end{array}$ & $\begin{array}{c}0.2[0.1-1.04]^{\infty} \\
(11)\end{array}$ \\
\hline $\begin{array}{c}\text { Dose of corticoseraids, } \\
\text { median [IQR] }\end{array}$ & $\begin{array}{c}20[10-457)^{\times 8} \\
(31)\end{array}$ & $\begin{array}{l}5[5.99)^{20 \times} \\
(20)\end{array}$ & $\begin{array}{l}3.75[0.5]^{* \infty} \\
(21)\end{array}$ & $\begin{array}{l}2.5[0.5]^{\infty 8} \\
(13)\end{array}$ & $\begin{array}{l}2.5[0.5)^{* x} \\
(11)\end{array}$ \\
\hline
\end{tabular}

Conclusions: TCZ therapy leads to a rapid and maintained improvement in patients with refractory GCA and/or with unacceptable side effects related to corticosteroids. However, the risk of neutropenia and infection should be kept in mind when using this biologic agent in patients with GCA.

Disclosure of Interest: None declared

DOI: 10.1136/annrheumdis-2017-eular.3262

\section{FRI0337 ANCA PATTERN IN GRANULOMATOSIS WITH POLYANGITIS AND MICROPOLYANGITIS. A RETROSPECTIVE ANALYSIS ON A MULTICENTRIC INCEPTION COHORT}

M. Felicetti ${ }^{1}$, R. Padoan ${ }^{1}$, S. Monti ${ }^{2}$, A. Berti ${ }^{3}$, G. Paolazzi ${ }^{3}$, G. Brunori ${ }^{4}$ R. Caporali ${ }^{2}$, F. Schiavon ${ }^{1} .{ }^{1}$ Department of Medicine DIMED, Operative Unit of Rheumatology, University of Padova, Padova; ${ }^{2}$ Department of Rheumatology, IRCCS Policlinico S.Matteo Foundation, University of Pavia, Pavia;

${ }^{3}$ Rheumatology Unit; ${ }^{4}$ Nephrology Unit, Santa Chiara Hospital, Trento, Italy

Background: Granulomatosis with polyangiitis (GPA) and microscopic polyangiitis (MPA) are multi-systemic diseases associated with anti-neutrophil cytoplasmic antibody (ANCA) often characterized by overlapping clinical manifestations. Recently ANCA pattern has been reported to define different clinical manifestations and long-term outcomes 1 .

Objectives: To analyze how ANCA could influence clinical manifestations and long-term outcomes in ANCA-associated vasculitis (AAV) patients, previously classified as GPA or MPA, from three different referral centers of Northern Italy. Methods: Clinical manifestations and long-term outcomes of GPA and MPA patients were retrospectively collected. We considered clinical (including BVASv3 and $\mathrm{VDI}$ ), radiological and histological data at diagnosis, relapse and mortality rates at the last follow-up. The complete cohort was splitted into three groups based on ANCA (anti-myeloperoxidase (MPO), anti-proteinase 3 (PR3), and ANCA negative) for statistical analysis.

Results: We included 171 patients, $90(52.6 \%)$ anti-PR3 positive, $52(30.4 \%)$ antiMPO positive and 25 (14.6\%) ANCA negative. Patients were mainly middle aged $(52.5 \pm 15.9$ years), Caucasian $(98.2 \%)$ and both sexes were equally represented (Female 53.2\%).

Anti-MPO positive patients were older (65 [52.5-69.7] years) at disease onset $(p<0.001)$, affected by more comorbidities $(p=0.045)$. They presented more frequently renal involvement $(p<0.001)$ with higher creatinine levels at diagnosis (0.98 [0.75-1.92] mg/dL, $p<0.001)$ (Fig. 1$)$, systemic symptoms $(p=0.039)$ but lower frequency of upper airways involvement $(\mathrm{p}=0.005)$.

Anti-PR3 positive scored higher BVASv3 at onset $(p<0.001)$ and presented more upper airways involvement $(p=0.033)$, lung nodules $(p=0.002)$ and skin purpura $(p=0.016)$.

ANCA negative showed a longer diagnostic latency (8.5 [3.5-49] months, $\mathrm{p}<0.0001)$, they presented with a higher VDI at diagnosis $(1[0-2.7], p<0.0001)$
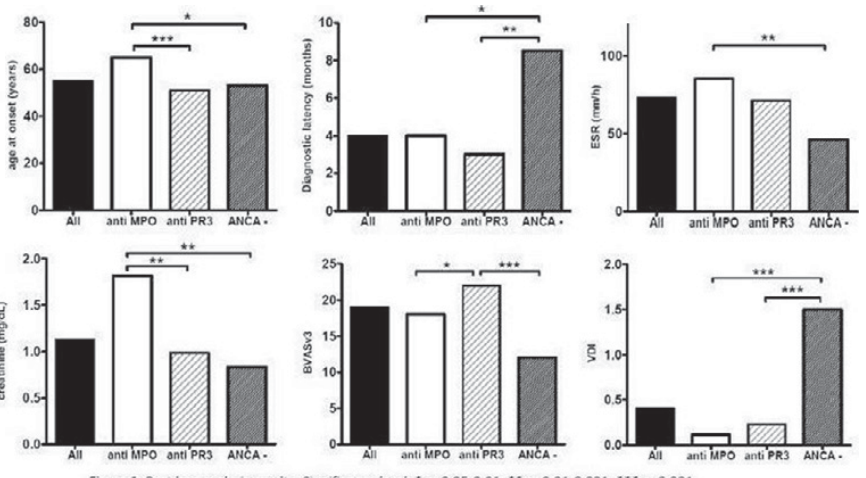
SR: eythrocyte sedimentation rate; BVASV3: Birmingham Vasculitis Activity Score version 3; VDI: Vasculitis Damage Index (VDI) 